\title{
A Population-Based Assessment of Emergency Department Observation Status for Older Adults With Cancer
}

\author{
Allison Lipitz-Snyderman, $\mathrm{PhD}^{\mathrm{a}}$; Adam Klotz, MD ; Renee L. Gennarelli, MSª; and Jeffrey Groeger, MD
}

\begin{abstract}
Background: Hospitals' use of observation status for patients with cancer presenting to the emergency department (ED) is not well understood. This model of care delivery may be a viable alternative to inpatient admission for patients with cancer presenting with certain conditions. Our objective was to assess the use of observation status among Medicare beneficiaries with and without cancer. Methods: Population-based SEER-Medicare data were used to assess differences in the use of observation status between Medicare beneficiaries aged $\geq 66$ years with and without cancer using a matched analysis $(n=151,183$ per cohort). We assessed the ratio of observation unit use to inpatient admission, between cancer and noncancer cohorts, and for patients diagnosed with breast, colon, lung, and prostate cancers. Poisson regression models were used to calculate observation rate estimates and $95 \% \mathrm{Cls}$ while adjusting for selected patient characteristics. Results: When considering the volume of hospitalizations, observation status is used less frequently among beneficiaries with cancer than those without (43 vs 69 observation status visits per 1,000 inpatient admissions, respectively). The estimated observation rate per 1,000 inpatient admissions was higher for beneficiaries aged $<75$ years versus those aged $\geq 75$ years, those with a Charlson comorbidity index of 0 vs 1 or $\geq 2$, and those without a prior hospitalization versus those with $\geq 1$ prior hospitalizations. Patients with breast and prostate cancers had higher adjusted and unadjusted observation rates per 1,000 inpatient admissions compared with those with colon and lung cancers. Conclusions: Observation status is used proportionately less for beneficiaries with cancer than those without. There may be opportunities to develop standards for ED staff to manage certain conditions for patients with cancer in observation status, and to reserve hospital resources for those who need it most.
\end{abstract}

For patients presenting to the emergency department (ED) with specific conditions such as chest pain, newonset atrial fibrillation, chronic obstructive pulmonary disease (COPD) exacerbation, and transient ischemic attack, observation status has been shown to be a viable alternative to inpatient admission. ${ }^{1-4}$ Observation status allows ED practitioners to make a more informed decision about whether to admit or discharge a patient

From a ${ }^{a}$ Center for Health Policy and Outcomes and ${ }^{\mathrm{b} D e p a r t m e n t ~ o f ~}$ Medicine, Memorial Sloan Kettering Cancer Center, New York, New York. Submitted January 23, 2017; accepted for publication May 19, 2017. Dr. Groeger has disclosed that he has a consulting or advisory role for T2 Biosystems. The remaining authors have disclosed that they have no financial interests, arrangements, affiliations, or commercial interests with the manufacturers of any products discussed in this article or their competitors.

This study was funded by Cancer Center Support Grant (P30 CA 008748). The funders had no role in this study design or conduct. based on additional diagnostic testing or response to a therapeutic intervention. Avoiding an inpatient admission is preferred by patients, especially because it reduces exposure to hospital-based risks and conditions. Typically, observation status is intended for $<24$-hours but can be extended if necessary. As hospitals experience increasing pressure to improve ED throughput and seek to minimize Medicare Recovery Audit Contractor

Author contributions: Study concept and design: Lipitz-Snyderman, Klotz, Groeger. Data acquisition, analysis, and interpretation: all authors. Statistical analysis: Lipitz-Snyderman, Gennarelli. Manuscript preparation: all authors. Critical revision for intellectual content: all authors. Administrative, technical, or material support: Lipitz-Snyderman, Gennarelli. Supervision: Lipitz-Snyderman, Groeger. Final approval of manuscript: all authors.

Correspondence: Allison Lipitz-Snyderman, PhD, Center for Health Policy and Outcomes, Department of Epidemiology and Biostatistics, Memorial Sloan Kettering Cancer Center, 485 Lexington Avenue, 2nd Floor, New York, NY 10017. E-mail: snyderma@mskcc.org 
penalties for inappropriate hospital admissions, observation status has become the focus of recent attention. ${ }^{5-7}$ Despite an increased focus on examining the use of observation status among the general population, its use among patients with cancer is not well understood. At the population level, observation status among older adults in general is extensive and increasing., ${ }^{5,8}$ This trend may reflect hospitals' recognition that some emergency care can be rendered without an inpatient admission. It may also reflect hospitals' response to changing reimbursement paradigms, because observation status might help reduce readmissions. The Hospital Readmissions Reduction Program, a provision in the Affordable Care Act, penalizes hospitals with higher-than-expected 30-day readmission rates for acute myocardial infarction, heart failure, pneumonia, total hip or knee replacement, and COPD. ${ }^{9}$ Of note, in a recent study, the authors found no difference in readmission rate with increased observation status use. This finding supports the notion that hospitals were not using observation status to avoid readmission penalties. ${ }^{10}$

Despite increasing attention on observation status, the extent to which it is used to successfully treat older adults with cancer is unknown. Patients with cancer aged $\geq 65$ years are hospitalized at a rate of 156.1 per 10,000 patients every year, which is greater than the rate for older adults who do not have cancer. ${ }^{11}$ Inpatient hospitalization is even higher for patients with terminal disease. ${ }^{12}$ Patients with cancer are frequently admitted to hospitals with an acute complication of their disease or its treatment, or for refractory symptoms. The specific clinical syndromes for which patients with cancer are seen in the ED is an active topic of investigation. Common complaints include pain, respiratory distress, and gastrointestinal issues. ${ }^{13}$ There may be opportunities to develop standards for ED staff to manage certain conditions for patients with cancer in observation status, and to reserve hospital resources for those who need it most.

In another study by our group, implementation of an observation unit at a Comprehensive Cancer Center was associated with a reduction in the overall rate of inpatient admission from the ED and true inpatient bed utilization. ${ }^{14}$ Other designated cancer centers are considering or have already implemented an observation pathway in their EDs. As we learn more from observation status at dedicated cancer centers, these lessons can be translated to commu- nity hospitals and vice versa. To better understand the opportunities that observation status may afford, we were interested in assessing the extent of observation status use among patients with cancer at a population level. We hypothesized that observation status without a follow-up inpatient admission occurs less often in patients with cancer than in the general population due to the complexity of care needs for this population. There may be opportunities to study its impact, potentially avoid unnecessary inpatient admissions, and maximize patient benefit.

\section{Methods}

\section{Data Source}

We used population-based SEER cancer registry data linked with Medicare claims to assess differences in the use of observation status between Medicare feefor-service beneficiaries with and without cancer, using a matched retrospective comparative analysis.

SEER, a consortium of population-based cancer registries sponsored by the $\mathrm{NCI}$, currently includes 18 registries covering approximately 28\% of the US population. For all incident cancers in their coverage areas, SEER registries collect information regarding site and extent of disease, the first course of cancerdirected therapy, and sociodemographic characteristics, with active follow-up for date and cause of death. Medicare claims have been linked to SEER records for adults aged $\geq 65$ years diagnosed with cancer and who reside in a SEER area. These claims include information on patient demographic characteristics as well as services, treatments, and procedures used by beneficiaries. Compared with the elderly US population, the SEER-Medicare population has a similar age and sex distribution, is somewhat more likely to live in urban and affluent areas, and has a smaller proportion of nonwhite individuals. The random 5\% sample of Medicare beneficiaries who reside in SEER regions was used to identify patients without cancer for the matched comparative analysis. ${ }^{15}$

\section{Study Sample}

Study cases included all fee-for-service Medicare beneficiaries aged $\geq 66$ years diagnosed with lung, breast, colorectal, or prostate cancer between 2006 and 2008, with follow-up through 2010. Beneficiaries $<66$ years of age were excluded due to lack of complete claims for the year before diagnosis. The 
Lipitz-Snyderman et al

month of diagnosis served as the study index date. Each Medicare beneficiary was followed for 2 years from diagnosis or until time of death, whichever came first. Patients with a prior cancer diagnosis, those whose cancer was diagnosed only at time of death, or those who were enrolled in an $\mathrm{HMO}$ or had incomplete Medicare Part A and B coverage from the year before diagnosis through the end of followup were excluded.

Cases were randomly matched to controls from the noncancer sample on a one-to-one basis without replacement by birth year range ( 5 years), geographic SEER region (Northeast, South, West, Midwest), sex, and race. Each matched control had the same study index date as its matched case. We excluded controls that were enrolled in an $\mathrm{HMO}$ or had incomplete Medicare Part A and B coverage from the year before the index date through the end of follow-up. Eligible controls had to be included in the data set for the duration of a case's follow-up period, because cases and matched controls were followed for the same duration.

\section{Variables and Outcomes}

Demographic and disease characteristics included age; race; a modified Charlson comorbidity index based on all inpatient, outpatient, and physician claims from 1 year before diagnosis ${ }^{16,17}$; and geographic region. For Medicare beneficiaries with cancer, we also included cancer type and disease stage.

Medicare outpatient claims were used to identify observation status for each beneficiary during the study period. To be classified as an "observation status," the revenue code for "general classification category" (0760) or "observation room" (0762) had to be present along with the procedure code for "hospital observation service per hour" (G0378) or "direct admission of a patient for hospital observation care" (G0379). 5,18 Visits were considered unique by date. Observation status visits that resulted in an inpatient admission were not identifiable and were therefore counted as an inpatient admission only.

Inpatient admissions were defined as any visit billed in MEDPAR files unique by date. Among the cases, we excluded admissions for cancer-directed surgeries because they were likely planned admissions (see supplemental eAppendix 1, available with this article at JNCCN.org). Prior inpatient admissions were also counted for each beneficiary for the year before the index date.

\section{Statistical Analyses}

We examined observation status with Medicare beneficiaries as the unit of analysis. We examined the frequency of inpatient admissions and observation status visits that did not result in an inpatient admission and calculated the average number of observation status visits and inpatient admissions per beneficiary throughout the study period. At the admission level, we used total observation status visits and total inpatient admissions to calculate the unadjusted rate of observation status visits per 1,000 inpatient admissions for the cancer (cases) and noncancer (controls) cohorts. This rate was also calculated separately for the first and second year from the index date for both cohorts. Poisson regression models were used to calculate observation rate estimates and 95\% confidence intervals while adjusting for patient characteristics, including prior hospitalization and comorbidities. Cancer-specific observation rate estimates were also adjusted for stage at diagnosis.

We received approval from the NCI for use of the SEER-Medicare data set for this analysis. This study was considered exempt research by the Memorial Sloan Kettering Cancer Center Institutional Review Board.

\section{Results}

A total of 161,457 cases met all eligibility criteria; of these, 151,193 (94\%) were successfully matched to a noncancer control. Demographic characteristics were similar between cohorts. The mean age at the study index date was 77 years and the median age was 76 years for both cohorts. Both cohorts were predominantly white $(85 \%)$, with $42 \%$ of beneficiaries residing in the Western SEER region (Table 1). The mean follow-up time was 18 months, with a median of 24 months among both groups.

\section{Overall Rate of Observation Status Visits}

There were 222,009 inpatient admissions and 12,934 observation status visits among the cancer cohort and 85,526 and 6,536, respectively, among the noncancer cohort. When considered in relation to inpatient admissions, the noncancer cohort had a higher rate of observation status visits per 1,000 inpatient admissions compared with the cancer cohort. After adjusting for patient characteristics, the cancer cohort had an estimated 43 observation status visits per 1,000 inpatient admissions compared with 69 per 


\section{Table 1. Matched Cancer Cohort Characteristics}

\begin{tabular}{|c|c|c|}
\hline & $\mathbf{N}^{\mathrm{a}}$ & $\%$ \\
\hline Total & 151,193 & - \\
\hline \multicolumn{3}{|l|}{ Sex } \\
\hline Male & 76,373 & 51 \\
\hline Female & 74,820 & 49 \\
\hline \multicolumn{3}{|l|}{ Race $^{a}$} \\
\hline White & 129,404 & 85 \\
\hline Black & 11,591 & 8 \\
\hline Other & 10,198 & 7 \\
\hline \multicolumn{3}{|l|}{ Geographic region ${ }^{a}$} \\
\hline Northeast & 31,834 & 21 \\
\hline West & 62,773 & 42 \\
\hline Midwest & 18,119 & 12 \\
\hline South & 38,467 & 25 \\
\hline \multicolumn{3}{|l|}{ Year of cancer diagnosis } \\
\hline 2006 & 51,914 & 34 \\
\hline 2007 & 50,808 & 34 \\
\hline 2008 & 48,471 & 32 \\
\hline \multicolumn{3}{|l|}{ Cancer type } \\
\hline Breast & 35,777 & 24 \\
\hline Colon & 29,827 & 20 \\
\hline Lung & 42,295 & 28 \\
\hline Prostate & 43,294 & 29 \\
\hline \multicolumn{3}{|l|}{ Stage at cancer diagnosis } \\
\hline 0 & 7,791 & 5 \\
\hline 1 & 28,664 & 19 \\
\hline 2 & 52,564 & 35 \\
\hline 3 & 21,471 & 14 \\
\hline 4 & 26,881 & 18 \\
\hline Occult ${ }^{\mathrm{b}}$ & 648 & 1 \\
\hline Unknown & 13,174 & 9 \\
\hline Mean (median) age at index date, y & $77(76)$ & - \\
\hline
\end{tabular}

${ }^{a}$ Noncancer control cohort has identical sex, race, and region distributions because they were matched along these characteristics. ${ }^{b}$ For patients with non-small cell lung cancer only.

1,000 inpatient admissions among noncancer controls $(P<.001)($ Table 2$)$

\section{Rates by Patient Characteristics}

The adjusted and unadjusted rates of observation status visits per 1,000 inpatient admissions differed by patient characteristics. Rates were higher for Medicare beneficiaries aged $<75$ years compared with $\geq 75$ years, those with a Charlson comorbidity index of 0 compared with 1 or $\geq 2$, and those without a prior hospitalization compared with $\geq 1$ prior hospitalizations (Table 2). Controls had a higher observation status visit rate compared with cases in both years 1 and 2 after the index date ( 58 vs 40 per 1,000 inpatient admissions in year $1 ; 72$ vs 52 per 1,000 inpatient admissions in year 2 ).

The higher rate of observation status visits per 1,000 inpatient admissions for patients without cancer compared with those with cancer remained after stratifying by prior inpatient admission. In fact, patients without cancer with $\geq 1$ prior hospitalization had a higher rate of observation status visits per 1,000 inpatient admissions than those with cancer and no prior hospitalizations ( 66 vs 47 , respectively).

\section{Rates Among Patients With Cancer}

Patients diagnosed with breast and prostate cancers had higher overall rates of observation status per 1,000 inpatient admissions compared with those with lung and colorectal cancers. In year 2 after diagnosis, the rate of observation status visits per 1,000 inpatient admissions was higher than in year 1 for colon, lung, and prostate cancers, but the opposite for breast cancer. Patients with breast cancer had an estimated $95 \mathrm{ob-}$ servation status visits per 1,000 inpatient admissions during the first year of follow-up, which is higher than the estimated rate of 58 observation status visits per 1,000 admissions for patients without cancer. Patients with cancer with lower-stage cancer at diagnosis had significantly higher estimated rates of observation status visits per 1,000 admissions $(P<.001)$.

\section{Discussion}

Observation status is a common alternative to inpatient admission for patients presenting to EDs. We found that observation status is used less among Medicare beneficiaries with cancer than those without.

We found that younger patients, those with no comorbidities, those with no prior hospitalizations, and those with lower stage at diagnosis had higher rates of observation status visits per 1,000 inpatient admissions. This suggests that observation status visits are more commonly used than inpatient admissions among patients who are relatively healthier at baseline, or that observation status visits are more likely to lead to inpatient admissions in sicker patients. We cannot discern between these scenarios from our data. Interestingly, we found high relative use of observation status visits among patients with breast cancer in year 1 , suggesting important differ- 
Lipitz-Snyderman et al

Table 2. Observation Status for Cancer and Noncancer Cohorts

\begin{tabular}{|c|c|c|c|c|}
\hline & \multicolumn{2}{|c|}{$\begin{array}{c}\text { Cancer Cohort } \\
(n=151,193)\end{array}$} & \multicolumn{2}{|c|}{$\begin{array}{c}\text { Noncancer Cohort } \\
(n=151,193)\end{array}$} \\
\hline & $\begin{array}{l}\text { Unadjusted Observation } \\
\text { Status Visits Per } 1,000 \\
\text { Inpatient Admissions }\end{array}$ & $\begin{array}{c}\text { Estimated Observation } \\
\text { Status Visits Per 1,000 } \\
\text { Inpatient Admissions } \\
(95 \% \mathrm{Cl})^{\mathrm{b}}\end{array}$ & $\begin{array}{l}\text { Unadjusted Observation } \\
\text { Status Visits Per } 1,000 \\
\text { Inpatient Admissions }\end{array}$ & $\begin{array}{c}\text { Estimated Observation } \\
\text { Status Visits Per 1,000 } \\
\text { Inpatient Admissions } \\
(95 \% \mathrm{Cl})^{\mathrm{b}}\end{array}$ \\
\hline \multicolumn{5}{|l|}{ Cohort } \\
\hline Total & 58 & $43(41,46)$ & 76 & $69(66,73)$ \\
\hline \multicolumn{5}{|l|}{ Age, y } \\
\hline$<75$ & 67 & $48(46,51)$ & 87 & $76(72,81)$ \\
\hline$\geq 75$ & 52 & $39(37,41)$ & 70 & $64(60,67)$ \\
\hline \multicolumn{5}{|l|}{ Sex } \\
\hline Male & 54 & $39(37,42)$ & 75 & $68(64,72)$ \\
\hline Female & 62 & $48(45,50)$ & 78 & $72(68,76)$ \\
\hline \multicolumn{5}{|l|}{ Race } \\
\hline White & 60 & $47(45,49)$ & 78 & $74(71,76)$ \\
\hline Black & 53 & $40(37,43)$ & 72 & $66(61,72)$ \\
\hline Other/Unknown & 48 & $43(40,48)$ & 64 & $69(62,78)$ \\
\hline \multicolumn{5}{|l|}{ Prior hospitalization } \\
\hline 0 & 63 & $47(45,50)$ & 84 & $74(70,78)$ \\
\hline$\geq 1$ & 47 & $40(37,42)$ & 64 & $66(62,70)$ \\
\hline \multicolumn{5}{|l|}{ Comorbidities } \\
\hline 0 & 69 & $52(49,55)$ & 92 & $82(77,87)$ \\
\hline 1 & 56 & $43(40,45)$ & 79 & $72(68,77)$ \\
\hline$\geq 2$ & 45 & $36(34,39)$ & 59 & $57(54,61)$ \\
\hline \multicolumn{5}{|l|}{ Geographic region } \\
\hline West & 57 & $47(44,49)$ & 73 & $69(65,73)$ \\
\hline Midwest & 58 & $48(45,52)$ & 84 & $81(75,87)$ \\
\hline South & 94 & $77(73,82)$ & 105 & $100(94,106)$ \\
\hline Northeast & 24 & $20(19,22)$ & 44 & $42(39,46)$ \\
\hline
\end{tabular}

annadjusted rates were calculated using total counts of observation status visits and inpatient admissions.

${ }^{b}$ Rate estimates and $95 \%$ Cls were calculated using Poisson regression models and adjusting for age, sex, race, prior hospitalization, comorbidities, and geographic region. Cancer-specific rate estimates were also adjusted for stage at diagnosis.

ences by cancer type. This finding could also similarly reflect the overall health status of the patient cohort. Alternatively, patients with breast cancer are often seen in the ED for complications of treatment administered in the adjuvant setting. As such, these patients may have a smaller burden of cancer-related symptoms than the other groups in this analysis.

There are several possible explanations for the lower use of observation status by Medicare beneficiaries with cancer. Patients with cancer may be more likely to require inpatient admission following care in an observation setting than those without cancer (33\% vs 20\%). ${ }^{14}$ This significant departure from general clinical experience may discourage ED practitioners from using observation status for their patients with cancer. We were not able to determine the patient's illness severity at the time of ED presentation. If patients with cancer presented with more severe complaints, it could explain the lower use of observation status. Older adult patients are also more likely to require skilled nursing care on discharge. Because the Centers for Medicare \& Medicaid Services policy requires 3 days of hospitalization for coverage, this may discourage ED practitioners from using observation status. ${ }^{19}$

There were some limitations in our study. In some cases, beneficiaries with cancer sought treatment at EDs for reasons unrelated to their cancer. We do not know this information from this data set. However, because we are comparing cohorts with 
and without cancer, the reasons unrelated to the cancer should be similar between groups. The true meaning of observation status varies by hospital. For some, it reflects a separate unit designed for monitoring patients and a unique management structure. For others, the term may be an administrative distinction. ${ }^{7}$ This inconsistency limits our ability to evaluate the impact of observation units on outcomes. We only included patients aged $\geq 66$ years, and our results may not be generalizable to patients of all ages. The study is limited to 2 years of treatment and may not reflect use for long-term survivors.

Moving forward, there may be opportunities to develop standards for ED staff to manage patients with cancer presenting with certain conditions and with different types of cancer. This can help optimize resource utilization in the ED. As newer management options for oncologic emergencies are identified, such as evolving risk stratification paradigms for febrile neutropenia, observation status will likely play an increasing role. ${ }^{20}$ The NCI has identified the management of oncologic emergencies as an important knowledge gap and facilitated the creation of the Comprehensive Oncologic Emergencies Research Network (CONCERN) consortium in March 2015 to address this need (https://epi.grants.cancer.gov/concern/). CONCERN is a research network comprising academic and community hospitals charged with describing the use of emergency care by patients with cancer and developing evidence-based management strategies that improve cancer outcomes. It will be important to isolate the conditions that should be managed in observation status, outside the hospital, or in the hospital, and to reserve hospital resources for those who need it most. Minimizing healthcare costs but also matching patient needs with the appropriate level and type of care should be a priority, especially given policy changes such as through the Medicare Access and CHIP Reauthorization Act of 2015 (MACRA) that incentivize high-quality and low-cost healthcare. ${ }^{21}$

\section{Conclusions}

Observation status is used less for Medicare beneficiaries with cancer than for those without. Future research is needed to understand the impact of using different models to care for patients with cancer with acute health issues on important patient health and cost outcomes, as well as key factors that contribute to the success of these models.

\section{References}

1. Gaspoz JM, Lee TH, Weinstein MC, et al. Cost-effectiveness of a new short-stay unit to "rule out" acute myocardial infarction in low risk patients. J Am Coll Cardiol 1994;24:1249-1259.

2. Willert C, Davis AT, Herman JJ, et al. Short-term holding room treatment of asthmatic children. J Pediatr 1985;106:707-711.

3. Bellew SD, Bremer ML, Kopecky SL, et al. Impact of an emergency department observation unit management algorithm for atrial fibrillation. J Am Heart Assoc 2016;5:pii: e002984.

4. Nahab F, Leach G, Kingston C, et al. Impact of an emergency department observation unit transient ischemic attack protocol on length of stay and cost. J Stroke Cerebrovasc Dis 2012;21:673-678.

5. Feng Z, Wright B, Mor V. Sharp rise in Medicare enrollees being held in hospitals for observation raises concerns about causes and consequences. Health Aff (Millwood) 2012;31:1251-1259.

6. Wiler JL, Ross MA, Ginde AA. National study of emergency department observation services. Acad Emerg Med 2011;18:959-965.

7. Baugh CW, Schuur JD. Observation care-high-value care or a costshifting loophole? N Engl J Med 2013;369:302-305.

8. Venkatesh AK, Geisler BP, Gibson Chambers JJ, et al. Use of observation care in US emergency departments, 2001 to 2008. PloS One 2011;6:e24326.

9. Joynt KE, Jha AK. Characteristics of hospitals receiving penalties under the Hospital Readmissions Reduction Program. JAMA 2013;309:342-343.

10. Zuckerman RB, Sheingold $S H$, Orav EJ, et al. Readmissions, observation, and the Hospital Readmissions Reduction Program. N Engl J Med 2016;374:1543-1551.

11. Anhang Price R, Stranges E, Elixhauser A. Cancer Hospitalizations for Adults, 2009. HCUP Statistical Brief \#125. February 2012. Agency for Healthcare Research and Quality, Rockville, MD. Available at https:// www.hcup-us.ahrq.gov/reports/statbriefs/sb125.jsp. Accessed September $20,2017$.

12. Rocque GB, Barnett AE, Illig LC, et al. Inpatient hospitalization of oncology patients: are we missing an opportunity for end-of-life care? J Oncol Pract 2013;9:51-54.

13. Mayer DK, Travers D, Wyss A, et al. Why do patients with cancer visit emergency departments? Results of a 2008 population study in North Carolina. J Clin Oncol 2011;29:2683-2688.

14. Lipitz-Snyderman A, Klotz A, Atoria CL, et al. Impact of observation status on hospital use for patients with cancer. J Oncol Pract 2015;11:7377.

15. Warren JL, Klabunde CN, Schrag D, et al. Overview of the SEERMedicare data: content, research applications, and generalizability to the United States elderly population. Med Care 2002;40(8 Suppl):IV-3-18.

16. Klabunde $\mathrm{CN}$, Potosky AL, Legler JM, Warren JL. Development of a comorbidity index using physician claims data. J Clin Epidemiol 2000;53:1258-1267.

17. Romano PS, Roos LL, Jollis JG. Adapting a clinical comorbidity index for use with ICD-9-CM administrative data: differing perspectives. J Clin Epidemiol 1993;46:1075-1079; discussion 1081-1090.

18. Feng Z, Jung HY, Wright B, Mor V. The origin and disposition of Medicare observation stays. Med Care 2014;52:796-800.

19. Lipsitz LA. The 3-night hospital stay and Medicare coverage for skilled nursing care. JAMA 2013;310:1441-1442.

20. Ahn S, Lee YS, Lee JL, et al. A new prognostic model for chemotherapyinduced febrile neutropenia. Int J Clin Oncol 2016;21:46-52.

21. Medicare program; Merit-Based Incentive Payment System and Alternative Payment Model Incentive Under the Physician Fee Schedule, and Criteria for Physician-Focused Payment Models. Federal Register Web site. Available at: http://www.federalregister.gov/a/2016-10032. Accessed August 10, 2017. 